\title{
Evolução a Longo Prazo e Complicações da Hipertensão Arterial após Transplante Cardíaco
}

\author{
Luiz Aparecido Bortolotto, Hélio Bernardes Silva, Edmar A. Bocchi, Giovanni Bellotti, \\ Noedir Stolf, Adib D. Jatene
}

São Paulo, SP

\begin{abstract}
Objetivo - Avaliar a evolução da hipertensão arterial (HA), e suas consequências, em pacientes submetidos a transplante cardíaco (TC) em uso de ciclosporina (CL).

Métodos - Em 65 pacientes submetidos a TC ortotópico, avaliamos a pressão arterial, creatinina sérica e níveis sangüíneos de CLnos períodos pré-operatório (15 dias antes do TC), pós-operatório precoce (15 e 30 dias) e tardio (6, 12 , 24, 48 e 60 meses); em 20 pacientes analisamos índice cardíaco e resistência vascular no pré, 15 e 30 dias, 6 e 12 meses após TC; em 33 pacientes, estudamos estrutura e função ventricular ao ecocardiograma, 24 \pm 13 meses após TC.

Resultados - Após 30 dias, a HA estava presente em $58,5 \%$ dos pacientes (50\% leve), enquanto na evolução tardia, a incidência da HA aumentou significativamente para $93 \%$ após um ano ( $85 \%$ moderada a grave). A creatinina sérica aumentou progressivamente do pré-TC $(1,43 \pm 0,5 \mathrm{mg} / \mathrm{dl})$ até após um ano $(1,83 \pm 0,9 \mathrm{mg} / \mathrm{dl})$, mantendo leve incremento até os 60 meses $(2,4 \pm 0,8 \mathrm{mg} / \mathrm{dl})$. Não houve correlação entre a HA, creatinina sérica e níveis de CL. $O$ índice cardíaco aumentou na fase precoce, enquanto a resistência periférica diminuiu no início e aumentou significativamente aos 12 meses. Ao ecocardiograma, $54 \%$ dos pacientes apresentavam hipertrofia de ventrículo esquerdo com função normal. Dos 31 pacientes que faleceram durante a evolução, dois tiveram a causa mortis diretamente relacionada a $H A$.

Conclusão - A HA em pacientes submetidos a TC em uso de CL ocorre precocemente, aumenta em prevalência e gravidade com o tempo e é mediada por aumento da resistência periférica, não se correlacionando com a nefrotoxicidade e com os níveis sangüíneos da CL, podendo agravar a insuficiência renal ou comprometer a longevidade do transplante, induzindo hipertrofia ventricular.
\end{abstract}

Palavras-chave: hipertensão, ciclosporina, transplante cardíaco

\section{Long-term Follow-up and Complications of Arterial Hypertension after Cardiac Trans- plantation}

Purpose - To evaluate the progression of arterial hypertension $(\mathrm{AH})$ and its consequences, in patients submitted to cardiac transplantation $(C T)$ in use of cyclosporine $(C Y)$.

Methods - In 65 patients submitted to orthotopic CT, we evaluated blood pressure, serum creatinine and blood levels of CY before, 15 and 30 days, and 6, 12, 24, 48 and 60 months after CT; in 20 patients we analyzed cardiac index and systemic vascular resistance pre-CT, 15 and 30 days, 6 and 12 months after CT; in 33 patients, we studied anatomic and functional modifications by echocardiography, 24 \pm 13 months after CT.

Results - Thirty days after CT, AH was present in $58.5 \%$ (50\% mild), and after one year, 93\% of patients were hypertensives ( $85 \%$ moderate-to-severe), remaining unchanged during the rest of follow-up. The serum creatinine progressively increased, reaching values significantly higher than those pre-CT after one year, persisting with a mild increment until 60 months. Echocardiography showed left ventricle hypertrophy in $54 \%$ of patients, all of which had normal function. Two patients died as a direct consequence of hypertensive complications.

Conclusion - $A H$ in patients submitted to $C T$ on $C Y$ use occurs early, increases in prevalence and severity during the follow-up and is mediated by an increase in vascular resistance. Also, the AH does not correlate to CY blood levels or nefrotoxicity, but it can impair renal function and compromise longevity of transplantation by inducing ventricular hypertrophy.

Key-words: hypertension, cyclosporine, heart transplantation

Arq Bras Cardiol, volume 69 (n 5), 317-321, 1997

Instituto do Coração do Hospital das Clínicas - FMUSP

Correspondência: Luiz Aparecido Bortolotto - Incor - Unidade de Hipertensão -

Av. Dr. Enéas C. Aguiar, 44 - 05403-000 - São Paulo, SP

Recebido para publicação em 4/12/96

Aceito em 16/9/97
A sobrevida de pacientes com insuficiência cardíaca refratária ao tratamento clínico é extremamente baixa, e, nos últimos anos, o transplante cardíaco (TC) tem sido uma opção terapêutica importante, modificando sobremaneira esta 
expectativa de vida ${ }^{1}$. A ciclosporina (CL), oligopeptídeo de origem fúngica com potente atividade imunossupressora ${ }^{2}$, foi marco importante na realização, tanto de TC quanto de outros órgãos, permitindo, além de maior sobrevida do enxerto, redução na morbidade, incidência de rejeição, período de hospitalização inicial e no número de reinternações ${ }^{3}$. No entanto, ao lado destes efeitos benéficos da droga, o uso crônico em grande escala da CL evidenciou efeitos tóxicos indesejáveis para a maioria dos transplantados, entre os quais destacam-se a nefrotoxicidade e a hipertensão arterial (HA) ${ }^{4,5}$. No homem, a HA é mais freqüente após TC ${ }^{6-9}$. Com efeito, estudos realizados em pacientes submetidos a TC demonstraram que o uso amplo da CL, como principal imunossupressor, associou-se com o aparecimento quase universal de $\mathrm{HA}^{10}$, contrastando com a menor prevalência, ao redor de $20 \%$, em pacientes tratados apenas com azatioprina e prednisona ${ }^{11}$. Analisando-se dados registrados até 1986 de 11 dos mais importantes centros em TC ${ }^{12}$, envolvendo um total de 367 pacientes, observou-se incidência variável de $45 \%$ a $92 \%$ de HA. Em nossa experiência, constatamos incidência de HA em $90 \%$ dos casos após um ano de $\mathrm{TC}^{13}$. A HA pode se manifestar sob formas graves, inclusive a maligna ${ }^{14}$, e, se não tratada, pode comprometer substancialmente a longevidade, por acelerar a aterosclerose coronária e, conseqüentemente, acarretar insuficiência cardíaca recorrente, retornando, assim, à condição clínica que indicou o TC. Os mecanismos envolvidos no desenvolvimento da HA induzida pela CL após TC não estão bem definidos. No entanto, a maioria dos dados da literatura ${ }^{15-18}$ sugerem que a gênese desta complicação é multifatorial e que todos os mecanismos envolvidos apresentam, como via final, o aumento da resistência vascular sistêmica $^{19}$.

No Brasil, não existem estudos demonstrando a evolução clínica e laboratorial da HA após TC e suas possíveis repercussões em órgãos-alvo, ou sobre a sobrevida desses pacientes. Assim, o objetivo deste trabalho foi analisar vários aspectos clínicos da HA e da insuficiência renal após TC, onde se destacam o acompanhamento evolutivo da HA per se, o papel da insuficiência renal, avaliação das alterações hemodinâmicas e avaliação das complicações cardíacas em 65 pacientes submetidos a TC em um período de 5 anos.

\section{Métodos}

De 1985 a 1991, 65 pacientes foram submetidos a TC ortotópico no INCOR com acompanhamento clínico pessoal, sendo que desse acompanhamento foram analisados o comportamento da pressão arterial (PA) e da função renal, correlacionando-as com os níveis séricos de CL. Além dessa análise, em 20 pacientes estudamos o comportamento hemodinâmico evolutivo durante um ano do período póstransplante, através de medidas de débito cardíaco e resistência vascular sistêmica. Também foi analisado em um subgrupo destes 65 pacientes o comportamento estrutural e funcional do ventrículo esquerdo (VE) do coração trans- plantado através de estudo ecocardiográfico. Finalmente, os pacientes falecidos nesse período de seguimento, foram submetidos, quando possível, a exame anatomopatológico, com análise mais detalhada do coração.

O grupo foi constituído de 55 homens e 10 mulheres, sendo 53 da raça branca, 10 da negra e dois da amarela. Quanto a etiologia da miocardiopatia que ocasionou a insuficiência cardíaca, encontramos 24 idiopáticas, 23 isquêmicas, quatro hipertensivas, duas reumatismais, cinco miocardites, dois peripartos e cinco chagásicas. Quatro pacientes apresentavam HA previamente a realização do TC. Para o controle adequado da PA utilizamos um a quatro medicamentos hipotensores, na maioria dos casos diuréticos, antagonistas dos canais de cálcio e simpatolíticos centrais. A dose de CL variou conforme o período após o transplante, sendo aparentemente maior no grupo precoce (até 30 dias) do que no período tardio.

Quanto ao comportamento da PA e função renal a longo prazo, a análise foi realizada observando-se dados referentes à $\mathrm{PA}$, níveis séricos de creatinina em $\mathrm{mg} / \mathrm{dl}$ e níveis sangüíneos de CL em mg/l, obtidos nos seguintes períodos pré-operatório (até 15 dias antes do TC), pós-operatório precoce (15 e 30 dias após transplante) e pós-operatório tardio $(6,12,24,48$ e 60 meses após o transplante). Todos os pacientes, excetuando-se 18 que evoluíram para o óbito precocemente, foram seguidos por um período mínimo de 24 meses. O nível sérico de CL foi determinado pelo método de radioimunensaio com anticorpo monoclonal ${ }^{20}$.

Para melhor análise do comportamento da PA, uma vez que vários pacientes faziam uso de medicação anti-hipertensiva, dividimos os pacientes em quatro grupos conforme classificação exposta na tabela I.

Em grupo de 20 pacientes do total de 65, realizamos análise periódica do débito cardíaco em $1 /$ min e da resistência vascular periférica em dynas $/ \mathrm{cm}^{-3}$, estudados por meio de cateter modelo Swan-Ganz por ocasião da biópsia endomiocárdia efetuada no período pré transplante, 15 dias, 30 dias, 6 meses e 12 meses após o TC.

Com relação à análise ecocardiográfica da estrutura e da função do VE após o TC, 33 pacientes foram estudados pela ecocardiografia bidimensional com Doppler através do aparelho Aloka, 24 \pm 13 meses após o TC, tendo sido analisadas medidas de septo interventricular em mm, diâmetro diastólico VE em mm, átrio esquerdo em mm, fração de ejeção, índice de massa VE em $\mathrm{g} / \mathrm{m}^{2}$ e relação volume/massa. Os critérios para hipertrofia de ventrículo esquerdo (HVE), seguiram as determinações da American Society of Echocardiography $^{21}$, ou seja, espessura de parede livre de VE $\geq 11 \mathrm{~mm}$ e/ou o índice de massa ventricular $>134 \mathrm{~g} / \mathrm{m}^{2}$ para homens e de $100 \mathrm{~g} / \mathrm{m}^{2}$ para mulheres.

Dos 65 pacientes analisados, 31 faleceram em diferentes períodos da evolução. Em 23, foi possível a determinação da causa mortis e a avaliação anatômica do coração através de exame anatomopatológico, observando-se peso do coração, a presença de hipertrofia e dilatação ventricular, e outras anormalidades do órgão transplantado. 


\section{Resultados}

Baseados na classificação da tabela I e na análise dos níveis de creatinina sérica e níveis séricos de CL obtivemos os resultados expostos na tabela II. Na fase de evolução precoce, já observamos o aparecimento de $\mathrm{HA}$ em 58,5\% dos pacientes após 30 dias, sendo a maioria de grau leve e de caráter lábil. Na evolução tardia, notamos um aumento progressivo do número de pacientes com $\mathrm{HA}$, atingindo incidência de $93 \%$ após um ano de evolução, com piora paralela da gravidade, isto é, com a maioria dos pacientes classificados como moderados e graves, mantendo-se estável daí para a frente, nos períodos de 24, 48 e 60 meses. De outra parte, os níveis séricos de creatinina aumentaram gradual e progressivamente após o transplante, atingindo valores significantemente superiores aos do pré transplante após um ano de evolução, mantendo-se com ligeiro incremento até os 60 meses. Apenas duas pacientes deste grupo evoluíram para insuficiência renal crônica terminal, necessitando tratamento dialítico, sendo uma delas submetida a transplante renal, com sucesso. Do ponto de vista estatístico, não se demonstrou correlação entre a HA e o aumento dos níveis de creatinina, indicando não haver correlação entre o desenvolvimento de HA e o déficit de função renal. Os níveis sangüíneos de CL foram mais elevados na fase precoce do transplante e a análise de regressão linear também não mostrou correlação entre os níveis sangüíneos de CL e os níveis de creatinina sérica ou com o aparecimento e gravidade da HA.

Os resultados do comportamento hemodinâmico são apresentados na tabela III. Observamos aumento do índice cardíaco e diminuição da resistência vascular sistêmica 15 e 30 dias após o transplante, embora esta última permaneça acima dos níveis considerados normais. Na avaliação de $6 \mathrm{e}$ 12 meses após a cirurgia, observamos aumento progressivo da resistência vascular sistêmica, com manutenção dos valores médios de índice cardíaco.

\begin{tabular}{|lcc|}
\hline $\begin{array}{c}\text { Tabela I - Classificação da hipertensão arterial baseada no nível da } \\
\text { pressão arterial diastólica e no número de medicações anti- } \\
\text { hipertensivas em uso, por ocasião da avaliação }\end{array}$ \\
\hline Grupo & $\begin{array}{c}\text { Pressão arterial } \\
\text { diastólica (mmHg) }\end{array}$ & $\mathrm{N}^{\text {o }}$ de drogas \\
\hline Normotenso & $<95$ & 0 \\
HA leve & $<95$ & 1 \\
& entre 95 e 104 & 0 \\
HA moderada & $<95$ & 2 \\
& entre 95 e 104 & 1 \\
HA grave & entre 104 e 119 & 0 \\
& $<95$ & 3 \\
& entre 95 e 104 & 2 \\
entre 104 e 119 & 1 \\
& $>119$ & 0 \\
\hline HA- hipertensão arterial. & \\
\hline
\end{tabular}

Os resultados da análise ecocardiográfica da estrutura e da função do VE são apresentados na tabela IV. Podemos observar que 18 (54\%) pacientes apresentavam HVE, todos com função ventricular sistólica normal (fração de ejeção $>0,60$ ). Nestes, o grau de hipertrofia pode ser considerado como leve. A HA foi mais importante no grupo de pacientes com HVE, onde $84 \%$ dos pacientes tinham graus moderado e grave, enquanto apenas $54 \%$ eram moderados e graves no grupo sem hipertrofia.

Como já mencionado, 31 pacientes faleceram em diferentes períodos da evolução, sendo que 23 foram submetidos a exame anatomopatológico. Exame macroscópico do coração mostrou peso do órgão variando de 300 a $900 \mathrm{~g}$, com HVE presente em 10 pacientes, sendo três leves, quatro moderados e três graves, e dilatação do VE em seis pacientes. A causa mortis foi diretamente relacionada a $\mathrm{HA} \mathrm{em}$ dois casos, uma por dissecção aguda de aorta e outra por cardiopatia hipertensiva.

\begin{tabular}{|lccccccc}
\multicolumn{7}{|c}{ Tabela II - Evolução da pressão arterial, níveis séricos de creatinina e de ciclosporina (NSCY) no período pré transplante e na evolução precoce (15 e 30 dias) } \\
e tardia (6, 12, 24, 48 e 60 meses) após o transplante cardíaco
\end{tabular}

HA- hipertensão arterial; NSCL = nível sérico de CY; *,** = p<0,05, * - comparação com o período pré-transplante cardíaco; ** - comparação com 15 e 30 dias e 6 meses. 


\begin{tabular}{|c|c|c|c|}
\hline \multicolumn{4}{|c|}{$\begin{array}{l}\text { Tabela III - Valores médios dos parâmetros hemodinâmicos, pressão } \\
\text { arterial média (PAM) em mmHg, índice cardíaco (IC) em } 1 / \mathrm{min} / \mathrm{m}^{2} \text { e } \\
\text { de resistência vascular sistêmica em dyn } / \mathrm{cm}^{3} \text {, obtidos em } 20 \text { pacientes } \\
\text { nos períodos pré-transplante, e durante a evolução (15 e } 30 \text { dias, } 6 \\
\text { meses, } 12 \text { meses e } 5 \text { anos) do transplante cardíaco }\end{array}$} \\
\hline & $\begin{array}{c}\mathrm{N}^{\circ} \\
\text { pacientes }\end{array}$ & $\begin{array}{l}\text { Índice } \\
\text { cardíaco }\end{array}$ & $\begin{array}{c}\text { Resistência Vascular } \\
\text { sistêmica }\end{array}$ \\
\hline pré-TC & 20 & $2,42 \pm 0,05$ & $1939 \pm 415$ \\
\hline \multicolumn{4}{|l|}{ pós-TC } \\
\hline 15 dias & 20 & $3,52 \pm 0,45^{*}$ & $1188 \pm 413^{*}$ \\
\hline 30 dias & 20 & $3,28 \pm 0,53$ & $1564 \pm 501$ \\
\hline 6 meses & 20 & $3,02 \pm 0,45$ & $1967 \pm 525 * *$ \\
\hline 12 meses & 20 & $2,91 \pm 0,5$ & $2033 \pm 399 * *$ \\
\hline
\end{tabular}

Tabela IV - Dados ecocardiográficos e do grau de hipertensão arterial em 33 pacientes, divididos conforme a presença ou não de hipertrofia ventricular esquerda (HVE)

\begin{tabular}{|c|c|c|}
\hline & $\begin{array}{c}\text { Com HVE } \\
\mathrm{n}=18(54 \%)\end{array}$ & $\begin{array}{c}\text { Sem HVE } \\
\mathrm{n}=15(46 \%)\end{array}$ \\
\hline \multicolumn{3}{|l|}{ Grau de hipertensão } \\
\hline Leve & $3(16 \%)^{*}$ & $7(46 \%)$ \\
\hline Moderada & $9(50 \%)$ & $6(40 \%)$ \\
\hline Grave & $6(34 \%)^{*}$ & $2(14 \%)$ \\
\hline Septo interventricular $(\mathrm{mm})$ & $12 \pm 0,9 *$ & $9,4 \pm 0,5$ \\
\hline Diâmetro diastólico VE (mm) & $48,6 \pm 5$ & $49,1 \pm 8$ \\
\hline Fração de ejeção & $72,2 \pm 8$ & $70,2 \pm 6$ \\
\hline Átrio esquerdo (mm) & $46,5 \pm 9$ & $41,6 \pm 9$ \\
\hline Índice de massa VE $\left(\mathrm{g} / \mathrm{m}^{2}\right)$ & $141,6 \pm 28 *$ & $109,3 \pm 26$ \\
\hline Relação volume/massa & $0,47 \pm 0,1$ & $0,57 \pm 0,1$ \\
\hline
\end{tabular}

\section{Discussão}

Tanto nossos dados como os da literatura ${ }^{4,710}$ permitem traçar um perfil bastante claro da HA associada ao uso de CL no TC. No Brasil, nosso estudo éo primeiro a mostrar que a HA em transplantados cardíacos, tratados com CL,é de aparecimento precoce e aumenta em prevalência e gravidade, em função do tempo, e que o seu aparecimento e progressão não guarda relação com o efeito nefrotóxico da droga. Estes dados confirmam achados previamente descritos na literatura internacional ${ }^{7,9,10,22}$, demonstrando o aparecimento precoce e progressivo da HA, após o TC, sem correlação com o aparecimento da insuficiência renal. Olivari e $\mathrm{col}^{7}$, de forma semelhante aos nossos dados, demonstraram, em 56 receptores de TC, a presença de HA em $68 \%$ dos pacientes, após duas semanas, e em $92 \%$, seis meses após o transplante, com níveis séricos de creatinina normais no início e elevados após 6 meses, não se relacionando esta elevação com a HA. Esta elevada incidência de HA, presente após o TC, é nitidamente superior a outros grupos de pacientes tratados com CL, isto é, pacientes com transplante de medula óssea ${ }^{23} \mathrm{e} \mathrm{renal}^{24}$, e pacientes não-transplantados com doenças auto-imunes ${ }^{25}$. Além disso, nenhum fator pré-operatório parece influenciar no desenvolvimento da HA desses pacientes ${ }^{26}$. Em nossa casuística, apenas seis pacientes submetidos ao TC apresentavam história prévia de HA. Contudo, esses indivíduos foram os que apresentaram as complicações hipertensivas mais graves, como dissecção de aorta, HVE grave com disfunção sistólica associada e hipertensão refratária, confirmando análise de outros autores onde os problemas clínicos mais graves, em decorrência da administração de CL, ocorrem naqueles previamente hipertensos ${ }^{15,18}$. Esta hipertensão necessita tratamento em 80 a $90 \%$ dos casos, e seu controle é difícil apesar do uso de múltiplos esquemas anti-hipertensivos ${ }^{9,18}$. Em nossa experiência, após um ano de evolução de TC, a maioria dos pacientes que desenvolveram hipertensão estavam sob uso de uma ou mais drogas anti-hipertensivas. Por sua vez, a insuficiência renal apareceu com maior significância a partir do $1^{\circ}$ ano, não mostrando correlação com o desenvolvimento da HA. No entanto, a presença de maior gravidade da HA a partir deste período pode estar influenciando a piora da função renal.

Os dados obtidos a partir da análise hemodinâmica sugerem que o aumento da PA, evolutivamente após o TC, está relacionado a um aumento da resistência vascular periférica, devendo ser conseqüência direta deste aumento. Em um estudo paralelo, que incluiu 12 pacientes, dos 20 pacientes aqui relacionados, mostrou que a resistência periférica mantém-se elevada após um ano, e, cinco anos após o TC os valores eram semelhantes aos observados com um ano de evolução ${ }^{26}$. Estes dados reforçam achados da literatura, em que estudos hemodinâmicos ${ }^{28,29}$ sugerem que a resistência vascular sistêmica elevada é a principal anormalidade hemodinâmica da HA, induzida pela CL, após o TC. Estas observações e a baixa incidência de HA previamente ao uso de $\mathrm{CL}^{11}$ contrariam as explicações iniciais ${ }^{17}$, segundo as quais o desenvolvimento da HA, após TC, seria conseqüência de uma elevação do débito cardíaco frente a uma resistência vascular inadequadamente elevada, em consequiência de insuficiência cardíaca crônica, prévia ao TC.

No que se refere às complicações hipertensivas, nossos dados de necropsia são os primeiros a se referir às complicações possivelmente relacionadas à HA. Como podemos verificar, em dois pacientes foi possível relacionar a causa do óbito com a HA, quais sejam dissecção aguda de aorta e miocardiopatia hipertensiva. Além disso, mesmo naqueles indivíduos em que o óbito não foi ocasionado diretamente por uma complicação hipertensiva, observa-se alterações anatômicas do VE induzidas pela HA, tal como a HVE (10 pacientes). A HVEé, reconhecidamente, complicação freqüente de indivíduos com HA essencial e é fator de risco independente para maior morbidade e mortalidade cardiovascular. Como nosso estudo demonstrou, a hiper- 
trofia pode ocorrer como complicação da HA após TC, mas outros fatores não relacionados à HA per se podem influenciar no desenvolvimento da mesma. Em outro estudo apresentado previamente ${ }^{29}, 14$ destes 33 pacientes realizaram ecocardiogramas periódicos, a partir do $15^{\circ}$ dia de pós-operatório, repetindo-se no $30^{\circ}$ dia, seis meses e 12 meses após o transplante. Nestes pacientes houve aumento significante da espessura de septo interventricular e de índice de massa ventricular após um ano de evolução, sem relação direta com o incremento de PA. Por outro lado, em ecocardiogramas periódicos realizados em 12 pacientes, pelo menos com quatro anos de evolução, constatou-se que a HVE aparece precocemente na evolução (por volta do $1^{\circ}$ mês), não se alterando significativamente na evolução mais tardia e não se correlacionando com o grau de hipertensão ${ }^{31}$. A maioria dos estudos em hipertensos, após TC, mostra a presença da HVE em diversos graus ${ }^{32,33}$. No entanto, a associação desta hipertrofia com a HA tem apresentado resultados conflitantes. Nossos dados e os obtidos por Farge e $\operatorname{col}^{32}$ não mostram nítida correlação, enquanto que em outro estu$\mathrm{do}^{33}$, envolvendo número pequeno de pacientes, os autores descrevem correlação direta entre o desenvolvimento da hipertrofia e a HA.

Resumindo, a elevação da PA em pacientes submetidos a TC em uso de CL, ocorre precocemente no curso do transplante, aumenta em prevalência e severidade com o decorrer do tempo e é mediada por aumento da resistência periférica. Esta HA não parece ser primariamente devida à nefrotoxicidade e não se correlaciona com os níveis sangüíneos da droga. No entanto, exige tratamento eficaz, pois pode agravar a insuficiência renal secundária à própria droga, ou mesmo, comprometer substancialmente a longevidade do transplante, por induzir hipertrofia ventricular e acelerar a aterosclerose coronária e, conseqüentemente, acarretar insuficiência cardíaca recorrente, retornando assim à condição clínica que indicou o TC.

\section{Agradecimentos}

Ao Dr. Alfredo Fiorelli, pela realização das medidas hemodinâmicas e ao Dr. Alvaro V. Moraes, pela realização dos ecocardiogramas.

\section{Referências}

1. Austen WG, Cosimi AB - Heart transplantation after 16 years. N Engl J Med ; 311: 1436-8.

2. Kahan BD - Cyclosporine. N Engl J Med 1989; 321: 1725-37.

3. Macoviak JA, Oyer PE, Stinson EB, Jamieson SW, Baldwin JC, Shumway NEFour-year experience with cyclosporine for heart-lung transplantation. Transplant Proc 1982; 17(suppl II): II-97-II101.

4. Curtis JJ - Cyclosporine-induced hypertension. In: Laragh JH, Brenner BM, eds - Hypertension: Pathophysiology, Diagnosis and Management. $1^{\text {st }}$ ed. New York: Raven Press, 1990: 1829-35.

6. Julien J, Farge D, Guillemain R et al - Hypertension artérielle chez le transplanté cardiaque. Presse Méd 1990; 19: 26-9.

5. Kaye M- The registry of the international society for heart and lung transplantation. Ninth official report-1992. J Heart Lung Transpl 1992; 11: 599-606.

7. Olivari MT, Antolick A, Ring WS - Arterial hypertension in heart transplant recipients treated with triple-drug immunossupressive therapy. J Heart Transplant 1989; 8: 34-9.

8. Starling RC, Cody RJ - Cardiac transplant hypertension. Am J Cardiol 1990; 65: 106-11.

9. Weidle PJ, Vlasses PH - Systemic hypertension associated with cyclosporine. A review. Drug Intell Clin Pharm 1988; 22: 443-50.

10. Thompsom MF, Shapiro AP, Johnsen AM et al - New onset of hypertension following cardiac transplantation: a preliminary report and analysis. Transplant Proc 1983(suppl 1); 15: 2573-7.

11. Thompsom MF, Shapiro AP, Johnsen AM et al - The contrasting effects of cyclosporin A and azathioprine on arterial blood pressure and renal function following cardiac transplantation. Int J Cardiol 1986; 11: 219-29.

12. Registry of the International Society of Heart Transplantation. Transplantation around the world. J Heart Transplant 1986; 5: 1-88.

13. Bortolotto LA, Bocchi E, Bernardes Silva $\mathrm{H}$ et al - Arterial hypertension after cardiac transplantation. Hypertension 1989; 13: 521.

14. Joss DV, Barret AJ, Kandra JR, Lucas CF, Desai S - Hypertension and convulsions in children recieving cyclosporin A. Lancet 1982; I: 906.

15. Luke RG - Mechanism of cyclosporine-induced hypertension. Am J Hypertens 1989; 4: 468-71.

16. Schachter M - Cyclosporine A and hypertension. J Hypertension 1986; 6: 511 6.

17. Starling RC, Cody RJ - Cardiac transplant hypertension. Am J Cardiol 1990; 65: 106-11.

18. Textor SC - De novo hypertension after liver transplantation. Hypertension 1993; 22: 257-67.

19. Scott JP, Higenbottam TW, Hutter JA, Large S, Wallwork J - Effects of the immuno- suppressant cyclosporine on the circulation of the heart transplant recipients. Am J Cardiol 1991; 67:628-632.

20. Ball PE, Munzer H, Keller HP, Abisch E, Rosenthaler J - Specific ${ }^{3} \mathrm{H}$ radioimmunoassay with a monoclonal antibody for monitoring cyclosporine in blood. Clinical Chem1988; 34: 257-61.

21. Sahn DJ, De Maria A, Kisslo J, Weyman A - Recommendations regarding quantitation in M-mode echocardiography: Results of a survey of echocardiographic measurements. Circulation 1978; 58: 1072-83.

22. Sturrock NDC, Lang CC, Struthers AD - Cyclosporin-induced hypertension precedes renal dysfunction and sodium retention in man. J Hypertension 1993; 11: 1209-16.

23. Gluckman E, Devergie A., Lokiec F- Use of cyclosporine for prevention of graftversus-host disease after allogenic bone marrow transplantation. In: Kahan BD, ed., Cyclosporine. Therapeuthic use in Transplantation. Philadelphia: Grunner e Stratton, 1988: 461-9.

24. European Multicentre Trial Group - Cyclosporin in cadaveric renal transplantation: One-year follow-up of a multicentre trial. Lancet 1983; II: 986-9.

25. Tindall RSA, Rollins JA, Phillips JT, Greenlee RG, Wells L, Belendiuk G - Preliminary results of double-blind, randomized, placebo-controlled trial of cyclosporine in myasthenia gravis. N Engl J Med 1987; 316: 719-24.

26. Gaiotto FA, Busnardo FF - Alterações hemodinâmicas no pós-operatório tardio de transplante cardíaco ortotópico. Rev Med 1994; 73: 31-8.

27. Ozdogan E, Banner N, Fitzgerald M, Musumeci F, Khaghani A, Yacoubi M-Factors influencing the development of hypertension after heart transplantation. J Heart Transplant 1990; 9: 548-53.

28. Greenberg ML, Uretsky BF, Reddy PS et al - Long-term hemodynamic follow-up of cardiac transplant patients treated with cyclosporine and prednisone. Circulation 1988; 71: 487-94.

29. Whitworth JA, Mills EH, Coghlan JP et al - The haemodynamic effects of cyclosporin A in sheep. Clin Exper Pharmac Physiol 1987; 14: 573-80.

30. Bortolotto LA, Bernardes Silva H, Bocchi E et al - Left ventricular hypertrophy in hypertensive heart transplant recipients. Hypertension 1991; 17: 411.

31. Graziosi P, Bortolotto LA, Fiorelli A et al - Comportamento da hipertensão arterial e repercussão no ventrículo esquerdo após transplante cardíaco. Arq Bras Cardiol 1993; 61: 137.

32. Farge D, Julien J, Amrein C et al - Effect of systemic hypertension on renal function and left ventricular hypertrophy in heart transplant recipients. J Am Coll Cardiol 1990; 15: 1095-101.

33. Angerman CE, Spes CH, Willems S, DominiakP, Kemkes BM, Theisen, K-Regression of left ventricular hypertrophy in hypertensive heart transplant recipients treated with enalapril, furosemide, and verapamil. Circulation 1990; 84: 583-93. 